\title{
THERMAL AND NONTHERMAL INTERPRETATIONS OF FLARE X-RAY BURSTS
}

\author{
S. KAHLER \\ American Science and Engineering, Cambridge, Mass., U.S.A.
}

\begin{abstract}
Various authors have presented arguments for either the thermal or the nonthermal interpretations of impulsive $E>20 \mathrm{KeV} X$-ray bursts and slowly varying $E<10 \mathrm{keV} \mathrm{X}$-ray bursts. In this review the arguments for and against the prevailing opinion that the impulsive bursts are nonthermal and the slowly varying bursts are thermal are presented.

For the impulsive bursts we discuss the spectra, electron mean free paths, center-to-limb distributions of both the numbers of events and spectra of events, and polarization data as relevant criteria. For the slowly varying events we discuss electron self collision times, distribution of X-ray temporal parameters, associated gradual rise and fall radio bursts, spectral and time profiles of special events and center-to-limb distributions of numbers of events as the relevant criteria.
\end{abstract}

\section{Introduction}

The prime goal of solar flare observations is to deduce the physical mechanisms which give rise to the release of a large quantity of energy in a short time. It is generally assumed that the magnetic field of the active region in which the flare occurs is the source of that energy and that the energy is released by some plasma process or processes (Sturrock, 1973). As the flare evolves, we can expect that the released energy will eventually manifest itself largely as heat. Observations late in the course of the flare should then be predominantly thermal in nature, but earlier in the event one may hope to observe phenomena of a nonthermal nature which will reflect the processes by which the energy conversion is carried out. In particular, the observations of certain kinds of X-ray events are thought by some to give good insights into the nonthermal phases of flares. Others have disagreed, arguing that these events are usually or always thermal in nature. In this paper we will discuss the different kinds of observed flare X-ray events and their properties and review the various interpretations given them in the literature.

In the interpretation of the X-ray spectra one must decide whether the electrons producing the emission are thermally relaxed or not. By thermally relaxed we understand that the electron distribution at any point in the X-ray source region has a Maxwellian velocity distribution and a well defined temperature. The temperature may vary from point to point and the ions may not have the same temperature due to the longer relaxation times (Spitzer, 1962). From the point of view of studying the nonthermal flare process the thermal interpretation is a pessimistic one since it says that from observations we can only hope to calculate the amount of energy converted to heat and the rate of that conversion. In the nonthermal point of view we are seeing $\mathrm{X}$-ray bursts from energetic electrons which are a direct product of the nonthermal process. Because the differences between the two interpretations are so critical to our 
understanding of flare phenomena it is important that we understand the bases for these two interpretations and their consequences.

\section{X-Ray Event Morphology}

The two kinds of X-ray events that we shall discuss have been described by Kane and Anderson (1970). An example is shown in Figure 1 (Kane, 1975). At high energies $(E>20 \mathrm{keV})$ the $\mathrm{X}$-ray event is impulsive, with a total time duration of about $1 \mathrm{~min}$, and has a relatively hard spectrum. The other component is seen best at low energies $(E<10 \mathrm{keV})$ and is characterized by a much longer time duration and a relatively soft spectrum. It is also closely associated with the main phase of the optical flare and is slowly varying in time. When it occurs, the impulsive event almost always takes place during the rising phase of the slow event. These two kinds of events have been widely

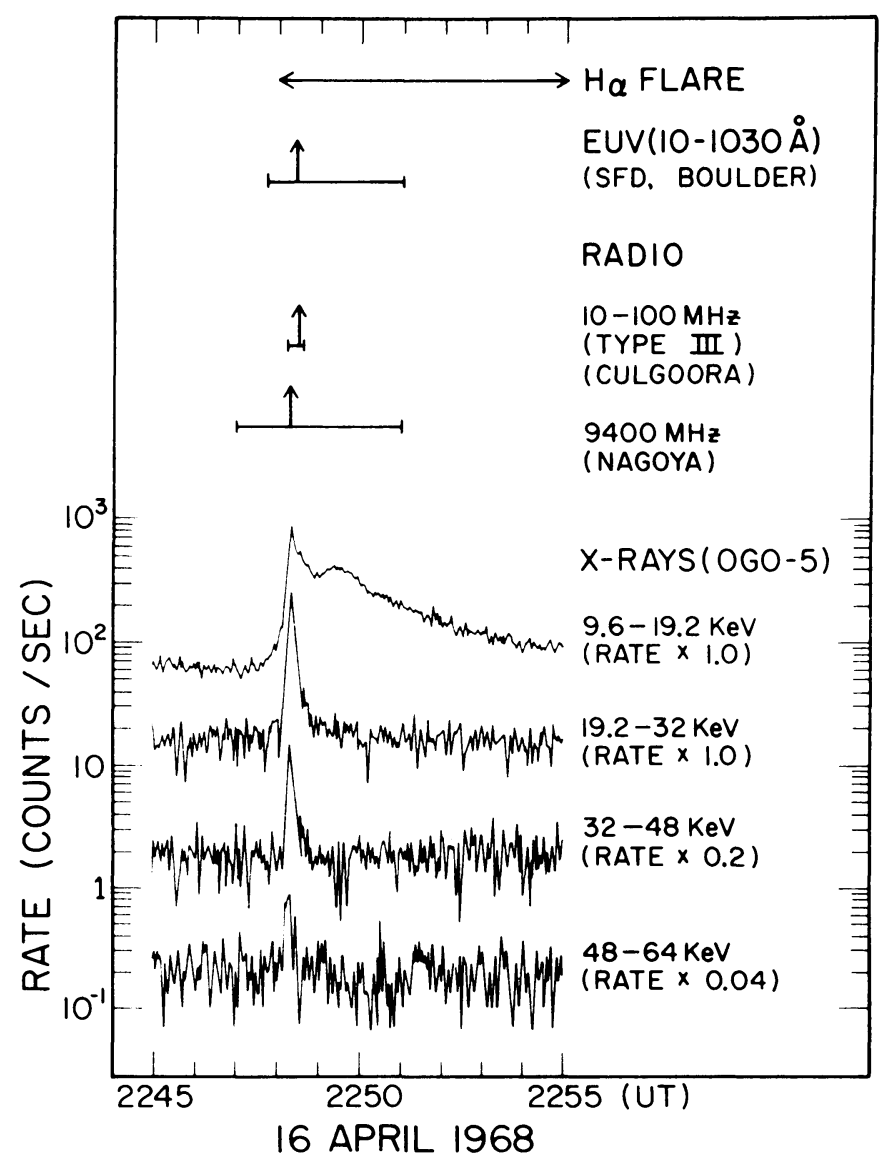

Fig. 1. The impulsive and low energy X-ray bursts observed with the OGO-5 scintillator (Kane, 1975). The impulsive burst is observed best at energies above $20 \mathrm{keV}$ and is well correlated with the impulsive microwave bursts, while the low energy component peaks later and has longer time constants. 
discussed in the literature, and various authors have argued thermal and nonthermal origins for each. In addition to these two kinds of events, a third class is known which is characterized by high energy events with relatively flat power law spectra out to $250 \mathrm{keV}$ or more and decay times on the order of minutes or tens of minutes. They are usually associated with type II or type IV radio bursts (Peterson et al., 1973; Frost and Dennis, 1971). Since everyone seems to agree that the latter events are nonthermal in nature, we shall consider thermal and nonthermal arguments only for the slowlyvarying and the impulsive events.

\section{High Energy $(E>10 \mathrm{keV})$ Events}

\section{(a) NonTHERMAL ARGUMENTS}

In their study of the properties of thirteen impulsive X-ray bursts Kane and Anderson (1970) advanced several reasons for choosing a nonthermal interpretation for the bursts. These were:

(1) The short rise time. For $\sim 40 \mathrm{keV} \mathrm{X}$-rays the $e$-folding rise times were $2-5 \mathrm{~s}$. The decay times were slightly longer at 3-10 s.

(2) The power law energy spectrum. They showed that a power law of the form:

$$
\mathrm{d} J / \mathrm{d} E=K E^{-\gamma} \text { photons } \mathrm{cm}^{-2} \mathrm{~s}^{-1} \mathrm{keV}^{-1},
$$

where $E$ is the photon energy and $K$ and $\gamma$ constants is a good fit to the observed X-ray spectrum at the peak of the event. They further showed that a bremsstrahlung interpretation of the X-ray spectrum yielded an instantaneous electron spectrum of the forn :

$$
\mathrm{d} J_{\mathrm{e}} / \mathrm{d} E_{\mathrm{e}}=A \cdot E_{\mathrm{e}}^{-\delta} \text { electrons } \mathrm{cm}^{-2} \mathrm{~s}^{-1} \mathrm{keV}^{-1},
$$

where $E_{\mathrm{e}}$ is the electron energy and $A$ and $\delta$ are constants. They concluded that the observations were not consistent with a thermal spectrum which can be represented by a single temperature. This is shown in Figure 2.

(3) The occurrence of the burst before the $\mathrm{H} \alpha$ maximum of the flare. The time of the $\mathrm{X}$-ray maximum preceded the $\mathrm{H} \alpha$ maximum by 0.5 to $3 \mathrm{~min}$.

These three arguments, especially the second one, have led most workers in the field to accept the nonthermal interpretation.

More recent work by Kane (1972) has tended to confirm this interpretation. He found a very close association between some impulsive X-ray bursts and type III radio bursts. Although only about one third of all impulsive X-ray bursts he observed are associated with reported type III bursts, it was found that when the X-ray and type III bursts are associated, the time correlation between the two emissions is very good. Figure 3 from Kane (1973b) shows an example of this close association. In general, not only the times of maxima of the bursts but also the total durations of the bursts were in close agreement. Since the type III radio bursts are produced by streams of nonthermal electrons (Lin et al., 1973) this close association again argues for the nonthermal interpretation of the impulsive X-ray bursts. A close temporal association also 


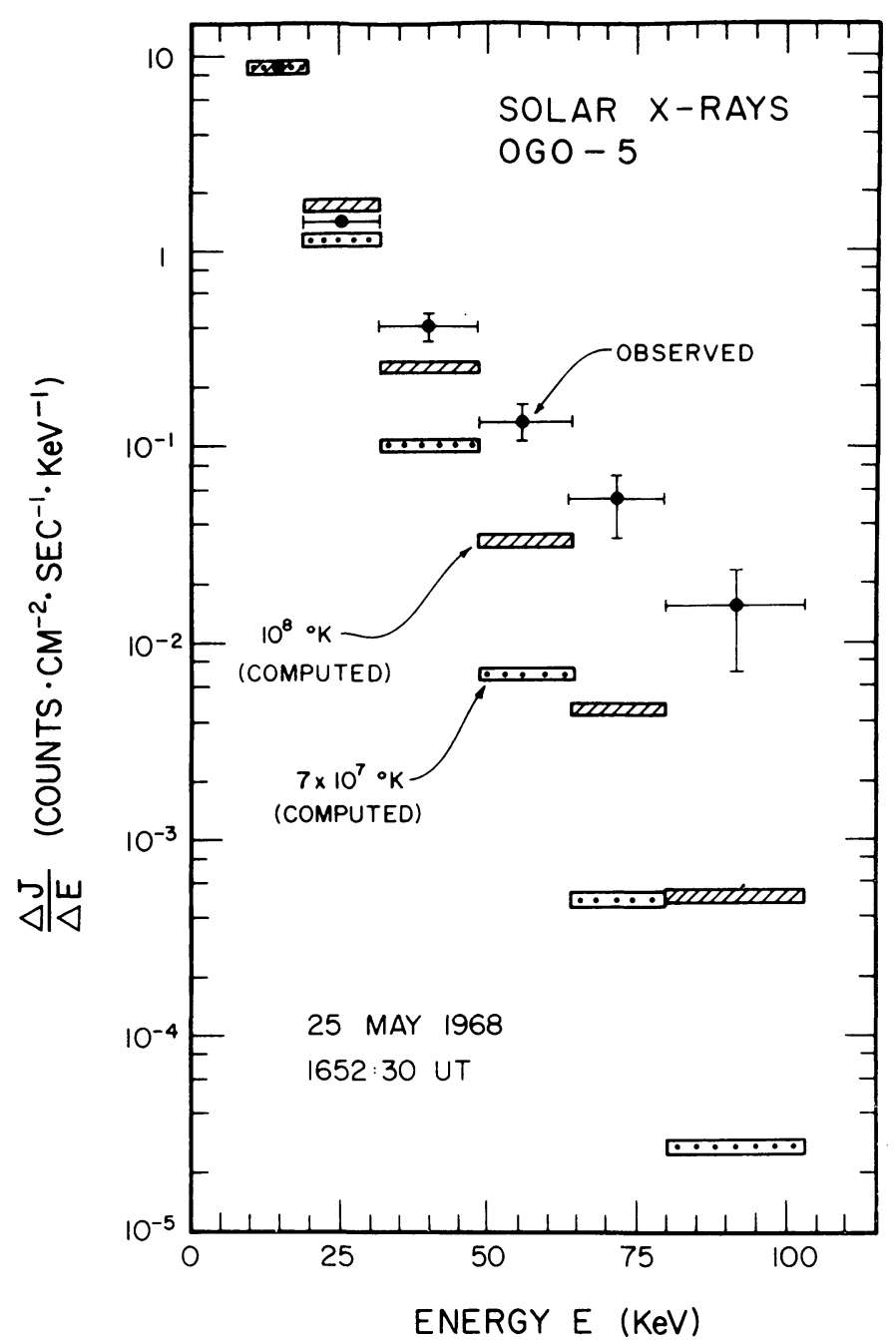

Fig. 2. The comparison of an impulsive burst spectrum compared with the calculated values of the spectra for plasmas with $T=7 \times 10^{7} \mathrm{~K}$ and $T=10^{8} \mathrm{~K}$. This shows the problem of trying to fit power law spectra with a single temperature thermal model (Kane and Anderson, 1970).

exists between the impulsive X-ray bursts and impulsive microwave bursts (Kane, 1972) which are believed to be due to gyrosynchrotron emission from nonthermal electrons (Ramaty, 1973). Kane (1973a) further found an approximate proportionality between the peak microwave flux and the peak X-ray flux in the correlated cases.

Another basis for the nonthermal interpretation is the observation of multiple or periodic impulsive X-ray bursts. One such burst was observed by Frost (1969) in 1969, March 1 and another burst with a 16 s periodicity was reported by Parks and Winckler (1969). In each case each individual burst was found to be correlated with impulsive microwave emission. 


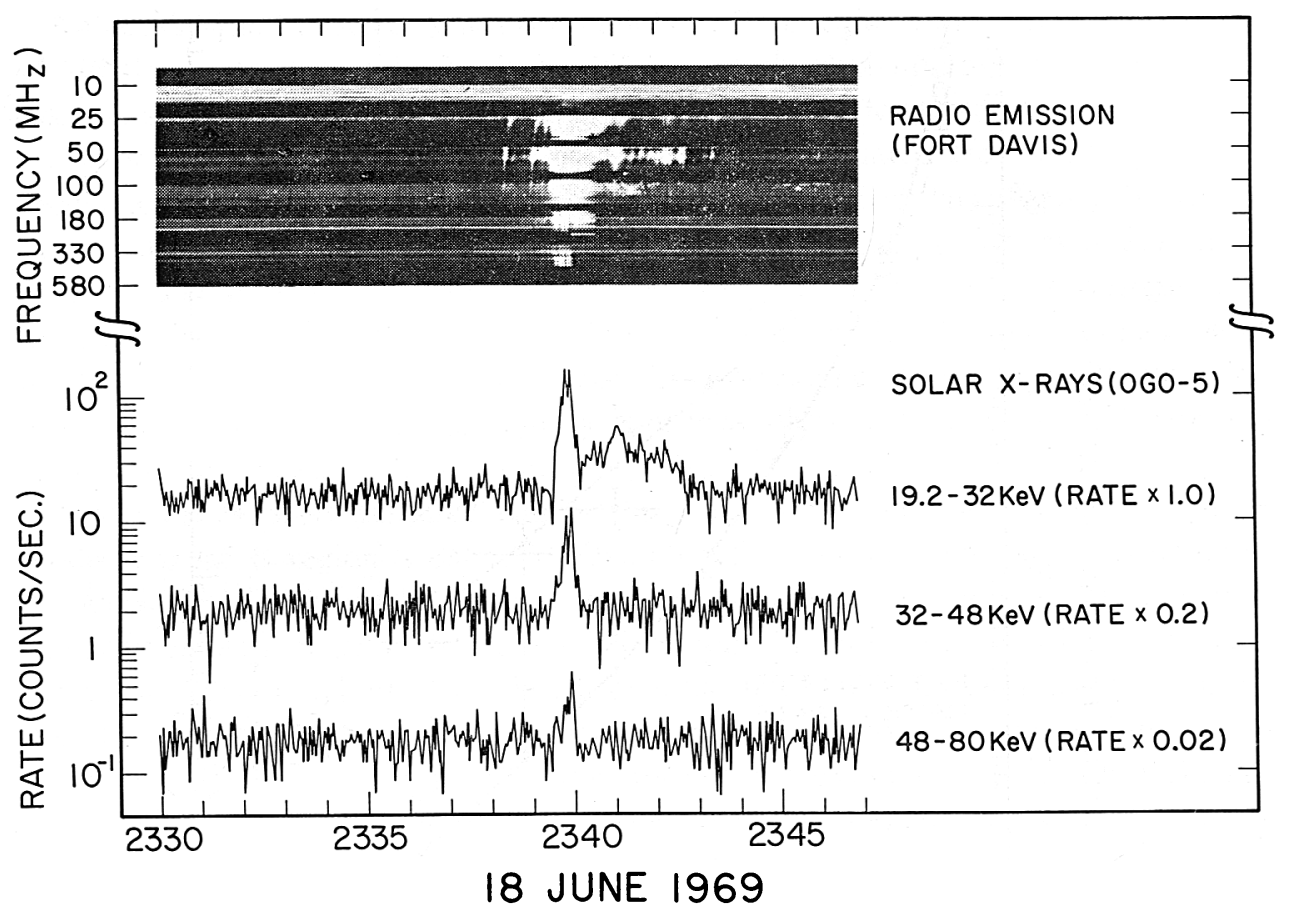

Fig. 3. An example of time correlated type III and impulsive X-ray bursts (Kane, 1973b).

\section{(b) THERMAL ARGUMENTS}

The general preference for the nonthermal interpretation is based largely on choosing the simplest interpretation of the observed spectrum. Since most impulsive bursts can be fitted by a simple power law energy spectrum but not a single temperature thermal spectrum, the nonthermal power law distribution is preferred. Chubb (1970), however, has discussed impulsive bursts in terms of a multithermal interpretation. He points out that X-ray flare measurements with crystal spectrometers have indicated the multithermal character of cooler flare plasmas and that it is only reasonable to expect this interpretation to be required at higher temperatures, i.e., shorter wavelengths. Chubb also points out that the impulsive bursts cannot be described by a simple power law over the entire observable energy range but instead they show a cutoff or steepening in the power law spectrum at $\sim 100 \mathrm{keV}$ which is characteristic of thermal emission. The thermal interpretation of impulsive bursts typically requires temperatures of $10^{8} \mathrm{~K}$ or more and emission measures of some $10^{3}-10^{4}$ times smaller than those characterizing cooler $\left(\sim 10^{7} \mathrm{~K}\right)$ plasmas observed with crystal spectrometers. Figure 4 shows his thermal interpretation of the 1969, March 1 impulsive event discussed by Frost (1969). The temporal behavior of the typical impulsive spectrum also is consistent with the thermal interpretation since it either first grows harder during the rise phase and then softens during the decay phase or it continuously 


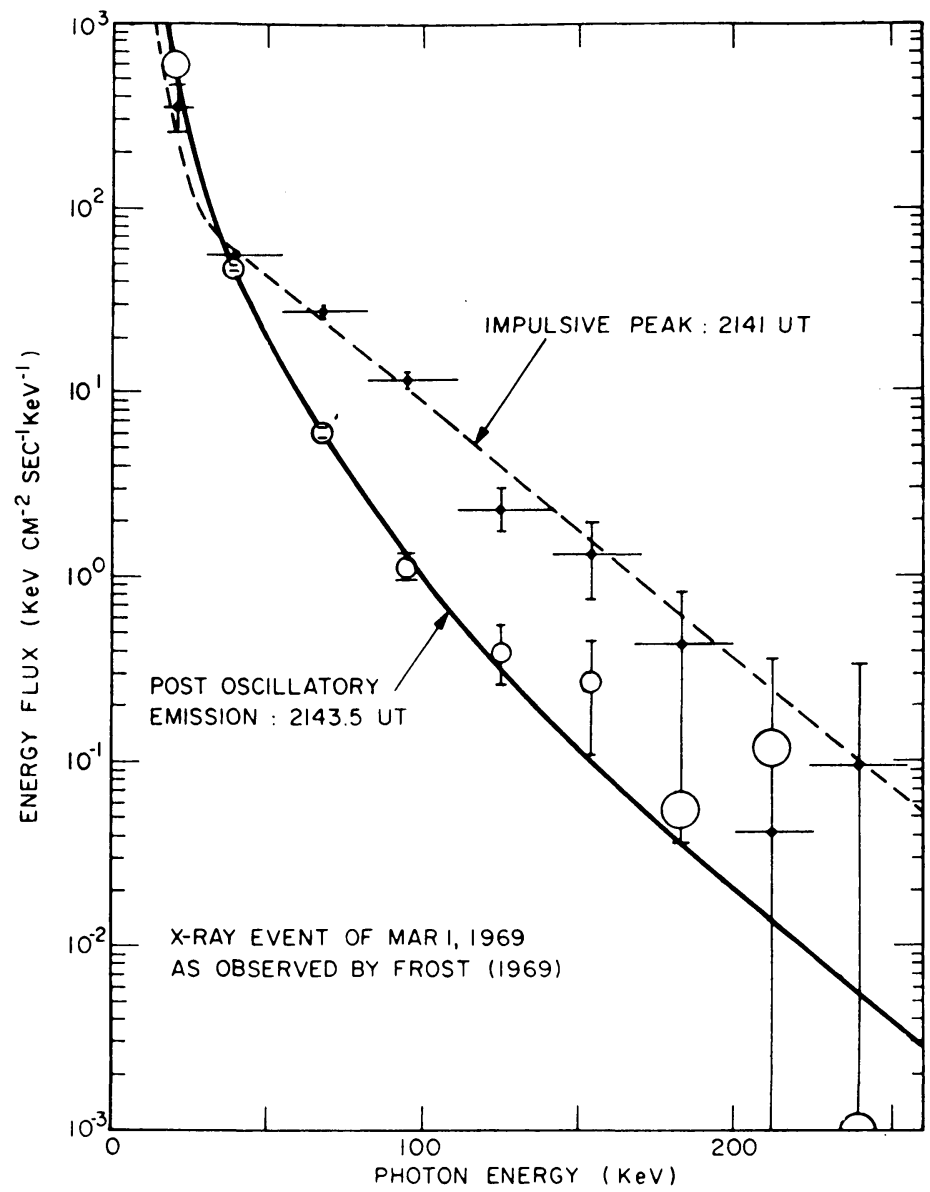

Fig. 4. The thermal interpretation by Chubb (1970) of an impulsive burst in 1969, March observed by Frost (1969). Chubb interprets the impulsive data at 2141 UT as free-free emission from a $3.7 \times 10^{8} \mathrm{~K}$ plasma.

softens throughout the burst (Datlowe et al., 1974) corresponding to a plasma which is heated and subsequently cools. Milkey (1971) similarly has pointed out that the impulsive X-ray bursts can be interpreted in terms of emission from a volume with a strong temperature gradient. In some cases the temperature distribution of the emitting region may result in a spectrum which mimics the power law non-thermal spectrum.

Brown (1974) has taken the next logical step in the multithermal analysis by deriving an analytic expression for the thermal source of any observed hard X-ray spectrum. For this he uses an emission measure $\mu(T)$ per unit temperature such that:

$$
\mu(T) \mathrm{d} T=n_{\mathrm{e}}^{2} \mathrm{~d} V .
$$

His result then yields $\mu(T)$ for any given observed X-ray spectrum. 
Kahler (1971a, b) used a simplified model of a flare region with a peak temperature of $10^{8} \mathrm{~K}$ to argue against Chubb's (1970) multithermal interpretation. Assuming electron streaming losses with no heat sources or sinks along the path and taking a range of emission measures given by Chubb, he calculated that the total energy needed to sustain the plasma against heat losses is prohibitively large for $n_{\mathrm{e}} \gtrsim 10^{9} \mathrm{~cm}^{-3}$. Lower electron densities require volumes larger than observational upper limits (Takakura et al., 1971). In addition, the mean free path of the electrons averaged over the $T \gtrsim 10^{8} \mathrm{~K}$ Maxwellian distribution is greater than the longest linear dimension of the flare volume, so that electrons are more likely to escape or be reflected by mirroring in a flux tube than to undergo binary collisions which will result in a Maxwellian velocity distribution.

Brown (1974) has countered Kahler's arguments by pointing out that his simple model of a $10^{8} \mathrm{~K}$ region is not valid. One must first unfold the actual distribution of the emission measure $\mu(T)$ per unit temperature and then, assuming constant pressure, calculate the thermal gradient as a function of temperature in order to calculate the conductive cooling at any point. Brown has used these calculations to obtain $\tau_{c}$, the characteristic time for the conductive temperature redistribution within the hot plasma. If this time is long, say $50 \mathrm{~s}$, then the entire decay of an impulsive burst can be explained by the conductive relaxation and the required energy input is small. If the time is short, say $2 \mathrm{~s}$, a much larger amount of energy is needed to sustain the burst. $\tau_{\mathrm{c}}$ is inversely proportional to $n_{10}^{3} \Sigma^{2}$ where $n_{10}$ is the density of the surrounding plasma in which $T=10^{7} \mathrm{~K}$ and $\Sigma$ is the cross section of the flux tube. A small change in either $n_{10}$ or $\Sigma$ results in a large change in $\tau_{c}$, and since these quantities are not well known, the values of $\tau_{c}$ are therefore very tentative. Brown has not answered one of the basic points raised by Kahler, that the mean free path of the electrons is excessively long. Brown calculates that for one large observed burst the scale distance for change in $T$ is comparable to the mean free path. This calculation was done only to decide whether the dominant cooling mechanism is conduction or electron streaming, but one must still consider whether the plasma can be thermally relaxed in the first place. Using Equation (8) of Takakura and Kai (1966) for the electron deflection time:

$$
t_{\mathrm{D}}=\frac{2.4 \times 10^{12} \varepsilon^{3 / 2}}{n_{0}} \mathrm{~s},
$$

where $\varepsilon$ is the ratio of the electron kinetic energy to its rest mass and $n_{0}$ the ambient density, we find a mean free path of

$$
1=v t_{\mathrm{D}} \approx 5 \times 10^{10} \mathrm{~cm}
$$

for a $50 \mathrm{keV}$ electron in a density of $n_{0}=10^{10} \mathrm{~cm}^{-3}$. This is on the order of one solar radius and more than an order of magnitude larger than the upper limit for an impulsive burst deduced by Takakura et al. (1971). Therefore, it still appears unlikely that the electrons will be thermally relaxed at densities of $10^{10} \mathrm{~cm}^{-3}$ or less. Avoiding this problem by assuming high densities then leads to the new problem of excessively high energy requirements in the treatments of both Kahler and Brown. Brown has pointed 
out that all the preceding arguments assume that the classical formulas for conductive cooling or electron streaming apply. If we have plasma turbulence then wave-particle interactions will constrict the heat loss and the thermal interpretation may then be valid.

\section{(c) ANISOTROPY AND POLARIZATION RESULTS}

If the impulsive bursts are produced by bremsstrahlung from nonthermal electrons, it is reasonable to expect that the bursts will be anisotropic in their distribution of emission and polarized due to the anisotropic nature of the electron velocity distribution. Pinter (1969) and Ohki (1969) each used the forty-six hard X-ray events listed by Arnoldy et al. (1968) to study the distribution of the events as a function of solar longitude. In each case the listed X-ray event was associated with an $\mathrm{H} \alpha$ flare which gave the position of the X-ray source on the disk. Ohki found a sharp limb darkening which could not be explained by the center-to-limb variation of $\mathrm{H} \alpha$ flares while Pinter found a peak in the longitude distribution at $40-50^{\circ}$. Pinter interpreted his results in terms of the model of Takakura and Kai (1966) in which electrons are trapped in and travel along a magnetic flux tube which is essentially parallel to the solar surface. The angular distribution function for bremsstrahlung (Sommerfeld, 1951) is

$$
J(\theta)=\sin ^{2} \theta(1-\beta \cos \theta)^{-4},
$$

where $\theta$ is the angle between the direction of motion of the electron and the direction of radiation and $\beta c$ is the electron velocity. Since higher energy bremsstrahlung peaks at smaller angles, the Takakura-Kai model and an east-west orientation for the magnetic field predicts that low energy bremsstrahlung will peak near central meridian and higher energy bremsstrahlung will peak closer to the limb, in accord with Pinter's findings. Pinter's calculations were improved by Elwert and Haug (1971) who used a power law distribution for electron energies and considered different pitch angles for the electrons. Their results were in qualitative agreement with Pinter's. Subsequently, Brown (1972) considered a model (de Jager and Kundu, 1963) in which electrons with a power-law distribution are continuously injected into a vertical magnetic field where they travel down into the chromosphere and rapidly decay in a thick target situation. Limb brightening is predicted, but the electron scattering in the thick target diminishes the center-to-limb effect, particularly for low energies. Phillips (1973) reanalyzed Ohki's data in terms of the de Jager-Kundu vertical field model by considering the distribution with respect to the Sun's apparent center and found the results to be consistent with a probability of occurrence which is independent of the distance from the Sun's center.

Kane (1973b) has analyzed the distribution of over 300 impulsive X-ray events observed on OGO 5 and finds that there is no significant center-to-limb variation in the frequency of occurrence of X-ray bursts associated with small solar flares. His data are shown in Figure 5. Datlowe et al. (1974) found a similar result using a smaller sample of events from their scintillation detector on OSO 7. These results probably 
present no difficulty to nonthermal electron models since non-vertical magnetic fields and various pitch angle distributions at the injection point of the de Jager-Kundu model (Brown, 1972) or departures from the east-west direction for the magnetic field of the Takakura-Kai model (Shaw, 1972) may substantially reduce any expected nonuniform distribution of events. For our purposes, however, it is important to note that Kane's result may not be consistent with a thermal origin of the impulsive bursts.

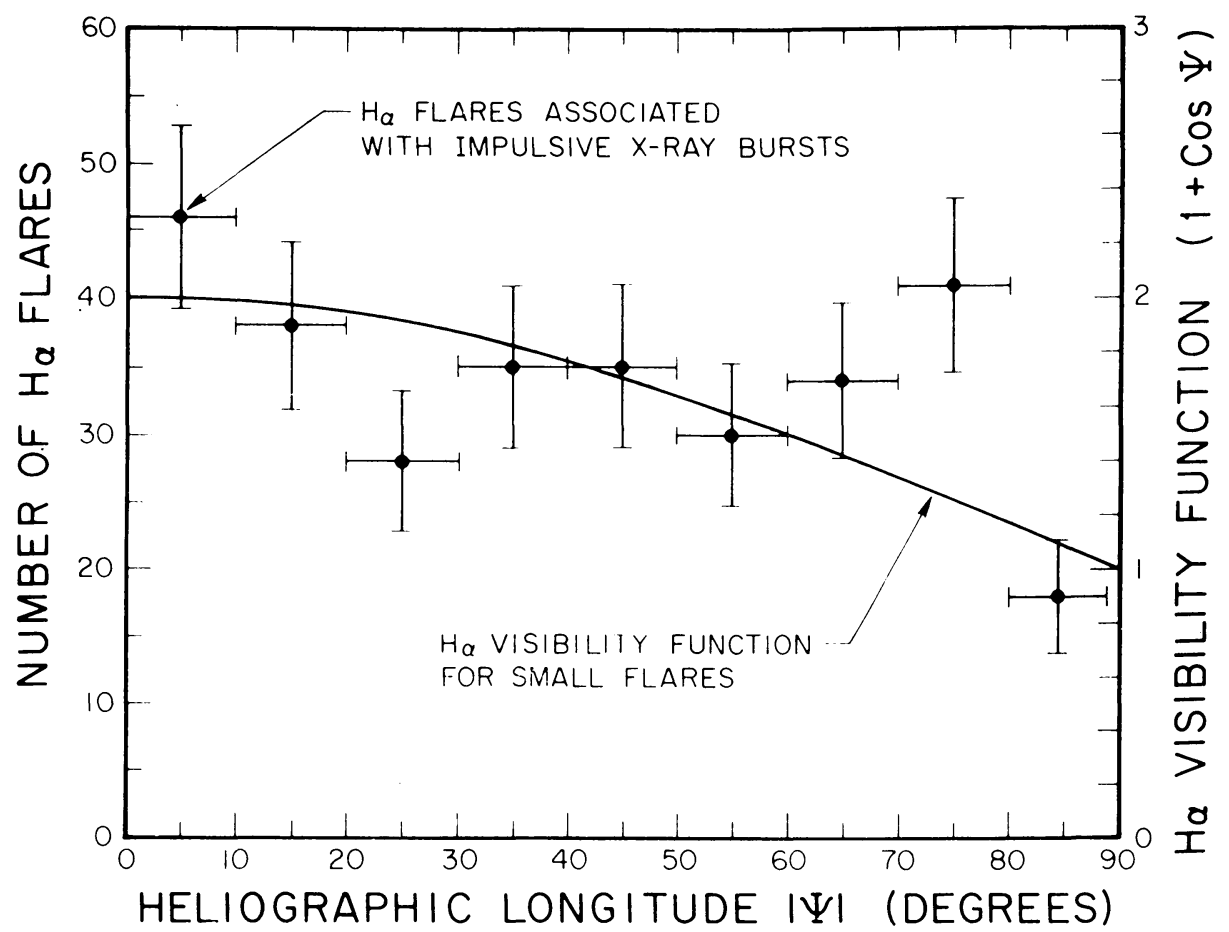

Fig. 5. The center-to-limb distribution of $\sim 300$ impulsive X-ray bursts measured by Kane (1973b). With the $\mathrm{H}$ r visibility function taken into account, the data are consistent with an isotropic source for the X-rays.

With an isotropic electron velocity distribution, no anisotropy in the X-ray emission is expected, but Santangelo et al. (1973) have shown the Compton backscattering from the solar surface can modify the intensity and spectral shape of the burst as observed at the Earth. Qualitatively, one expects some limb darkening, but the quantitative value depends on the energy spectrum and the effective passband of the X-ray detector.

In a recent result Datlowe et al. (1974) found an asymmetric distribution of X-ray burst spectral indices with solar longitude. Figure 6 shows how the average spectral index of a burst, $\bar{\gamma}$, becomes larger for limb and over-the-limb events. According to the authors, this result is statistically significant at the $99 \%$ confidence level and is too large to be explained by the center-to-limb variation of spectral hardening by Comp- 


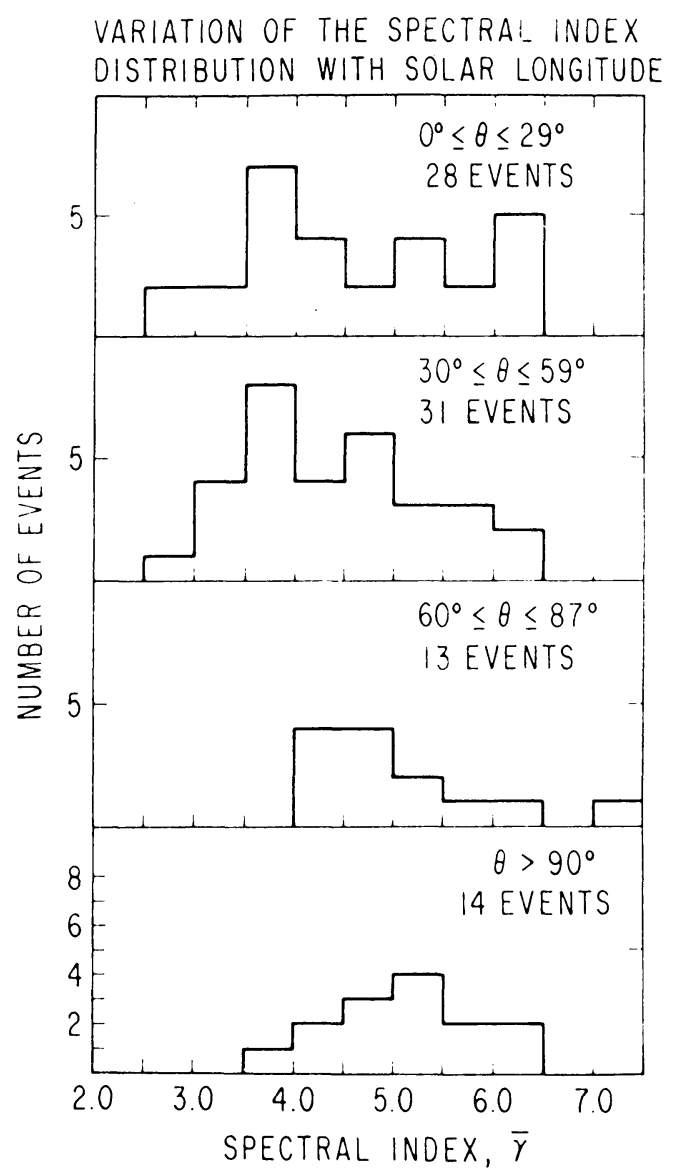

Fig. 6. The variation with solar longitude of the distribution of the average spectral indices for impulsive events measured by Datlowe et al. (1974). Events in the top three panels are identified with $\mathrm{H} \alpha$ flares or subflares while events in the bottom panel are from unreported events believed to be from over the limb.

ton back-scattering as discussed by Santangelo et al. (1973). They attribute the variation to directivity of the electron pitch angle distribution in the solar magnetic fields. Their result certainly appears to conflict with the thermal interpretation.

The polarization observations of flares reported by Tindo and his colleagues (Tindo et al., 1972a, b; 1973) have provided one of the strongest arguments for the nonthermal interpretation of hard X-ray bursts. Polarimeters consisting of beryllium scattering blocks and three pairs of counters were flown on the Intercosmos 1, 4 and 7 satellites to measure the polarization and polarization angles of flare $\mathrm{X}$-rays. The Intercosmos 4 instrument was used to determine these parameters for two flares in 1970, October 24 and November 5 (Tindo et al., 1972b). The maximum polarization of the two events was 0.16 and 0.21 respectively and in each case the plane of polarization is found to be near the plane of the projected radius vector of the flare on the 
solar disk. Tindo et al. (1972b) compared the flux profile of their broad band counters with a scintillation counter on board and found the closest correlation with the $15 \mathrm{keV}$ energy channel. The fact that a significant polarization has been measured for a number of hard X-ray bursts is not a conclusive argument against the thermal interpretation since some polarization will be produced by the albedo X-rays from flares (Santangelo et al., 1973). The reflected radiation will be linearly polarized along a line perpendicular to the projected radius vector of the flare on the disk rather than parallel to it as found by Tindo et al. Brown et al. (1974) have criticized the method of calibrating the polarization data by assuming a zero polarization late in the event on the grounds that some residual polarization will always be present in the albedo flux. Further polarization measurements of flares, especially those near sun center where the reflected radiation should be unpolarized and those near the limb where the plane of the reflected radiation should be parallel to the limb, could determine the validity of the thermal interpretation.

An additional problem from the point of view of Chubb's (1970) interpretation is whether the large X-ray flares observed by Tindo et al. correspond to a larger scale of

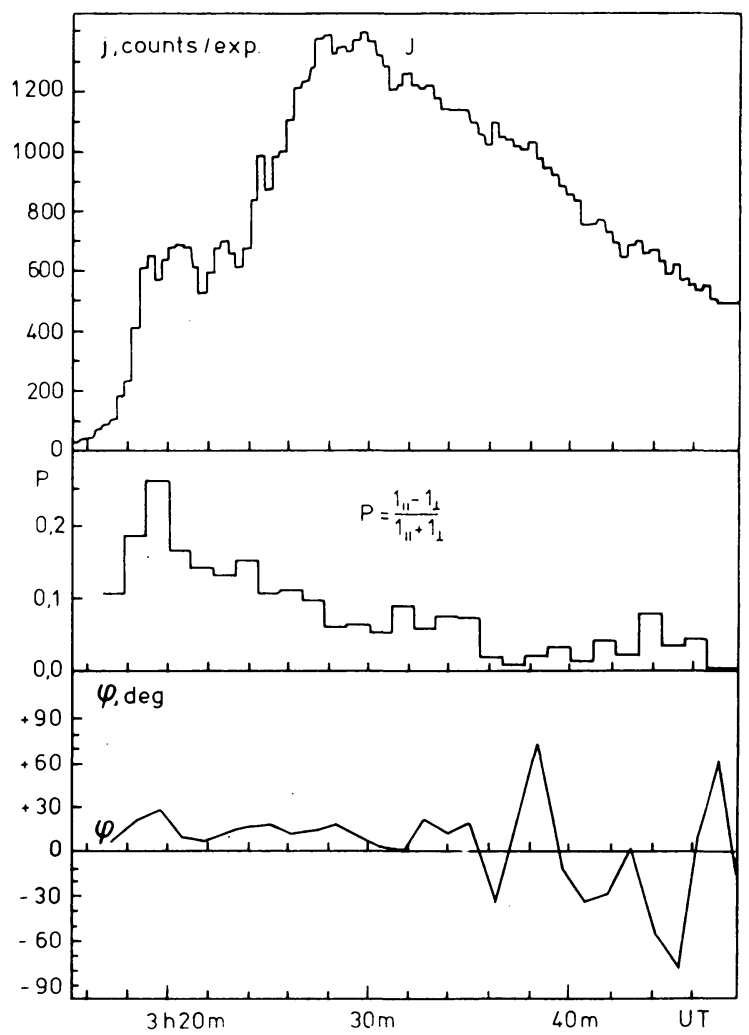

Fig. 7. The measurement of linear polarization by Tindo et al. (1972a) of a flare in 1970, November 5 .

(a) is the flux of the $E \sim 15 \mathrm{keV} X$-rays; (b) is the degree of polarization; and

(c) is the angle of polarization. 
the impulsive bursts of Kane and Anderson (1970) and Frost (1969) with a characteristic spectral cutoff at $\sim 100 \mathrm{keV}$ or whether they are more like the second phase of the two phase events reported by Frost and Dennis (1971). The event of 1970, November 5 , is shown in Figure 7. The facts that the flares observed on Intercosmos 4 were large $\mathrm{H} \alpha$ events, with polarization persisting for 5-10 min, and that the existence of a spectral cutoff around $100 \mathrm{keV}$ is in question, suggest the possibility that these may not be the impulsive events which Chubb interpreted as thermal.

\section{Low Energy Events}

\section{(a) THERMAL ARGUMENTS}

In contrast to the impulsive X-ray bursts, the low energy $(E<10 \mathrm{KeV}) \mathrm{X}$-ray events are almost always treated as thermal in origin. This is due in large part to the analysis of data from Bragg crystal spectrometers in which flare spectra can be observed with high wavelength resolution from $\sim 0.6$ to $\sim 20 \AA$ (Doschek et al., 1972). Temperatures may be estimated by (1) comparing the ratios of resonance lines of hydrogenic ions to helium-like ions of the same element; (2) measurement of the slope of the continuum; and (3) measuring the ratio of satellite lines due to dielectronic recombination to resonance lines due to collisional excitation. The temperatures obtained by these methods range from $\lesssim 10^{7} \mathrm{~K}$ to $3.4 \times 10^{7} \mathrm{~K}$. The various methods used generally give varying temperatures for the same event, indicating that a multithermal origin exists for the flare plasma.

Kahler et al. (1970) discussed two X-ray flares observed with a proportional counter on OGO 5 and argued that the $E<10 \mathrm{keV}$ radiation was thermal in origin because (1) the flux-time profiles of their detector were slowly varying and not impulsive as would be expected from a comparison with the high energy impulsive events; and (2) for a flare density of $n_{\mathrm{e}} \sim 10^{9}-10^{10} \mathrm{~cm}^{-3}$ the self collision time, i.e., the time for binary collisions to relax any deviation from a Maxwellian velocity distribution, is on the order of $10^{-1}$ to $10^{\circ} \mathrm{s}$. These arguments are not conclusive for the following reasons. First, the time scales of low energy nonthermal bursts, if they exist, do not have to be similar to those of the high energy bursts. Kane and Anderson (1970) found that the rise times of the impulsive events were longer for lower energies. In addition, there are numerous low energy bursts that have much shorter time scales than the two events presented by Kahler et al. (1970). For example, Culhane and Phillips (1970) discussed an event observed in 1968, January 23 that lasted only $4 \mathrm{~min}$. The problem with Kahler et al.'s second argument involving self collision times is that it is model dependent; it assumes a volume of energetic electrons which interact among themselves and have lifetimes much greater than the self collision time for relaxation. One could postulate a thick target model of continuous injection similar to that suggested for impulsive bursts (Brown, 1972). In such a case the electrons are nonthermal with lifetimes much shorter than the X-ray burst duration and comparable to or less than the self collision time.

Drake (1971) has statistically analyzed over 4000 solar X-ray bursts using data 
from the 2 to $12 \AA$ Geiger counter experiments on Explorers 33 and 35. He plotted the differential distributions of the $\mathrm{X}$-ray bursts with respect to rise time, decay time, total duration, ratio of rise time to total duration, and ratio of rise time to decay time. Figure 8 from his paper shows the distribution of the ratios of the rise time to the decay time. In this distribution, as in the others, there is no evidence for the existence of more than one class of X-ray burst in the $2-12 \AA(1-6 \mathrm{keV})$ range. More classes may exist but cannot occur for a substantial number of bursts. For the great majority

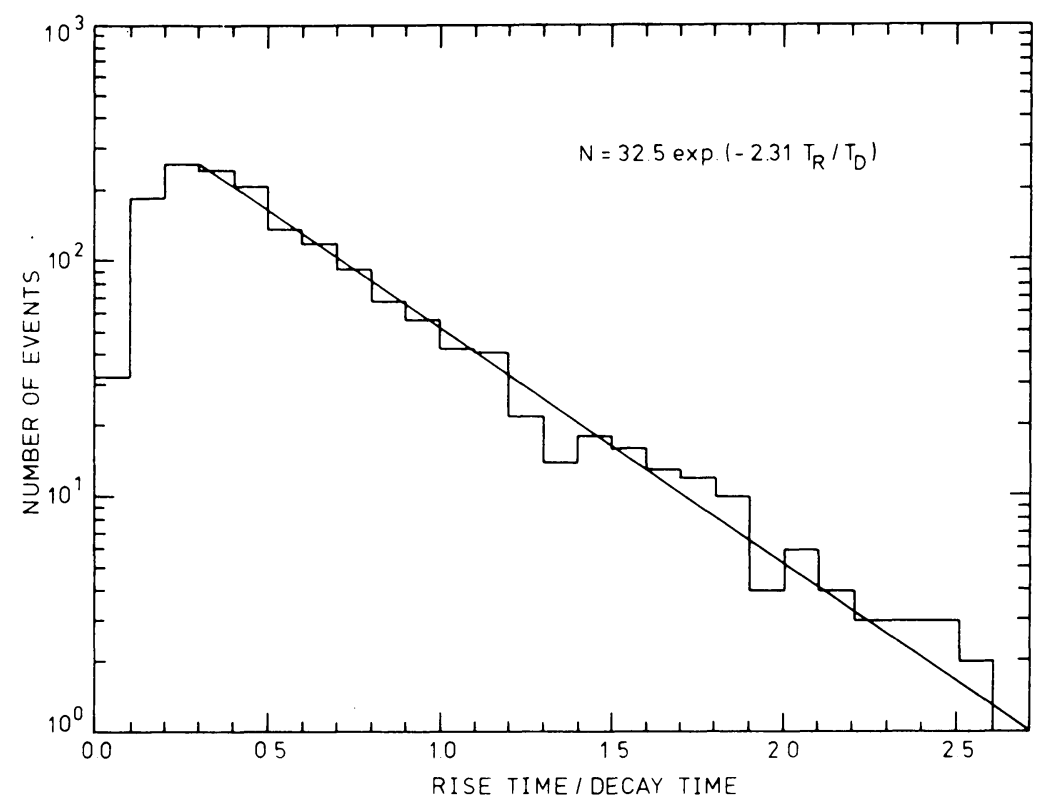

Fig. 8. The differential distribution of 2-12 $\AA$ X-ray bursts with respect to the ratio of the rise time to the decay time (Drake, 1971). The straight line is a least-squares fit.

of soft X-ray bursts then we can conclude that (1) they are all thermal or ((2) they are all nonthermal or (3) some are thermal and some are nonthermal but the time constants characteristic of each are similar.

Hudson and Ohki (1972) used the temperatures and emission measures derived from sixteen X-ray flare events observed with broad band detectors on the Solrad and Vela satellites to calculate the intensity of the associated 16 or $17 \mathrm{GHz}$ microwave event assuming an isothermal plasma. The measured intensity of the 'gradual rise and fall' or 'post burst increase' radio event near the time of the maximum X-ray emission measure was compared to the calculated value and good agreement was found. Hudson and Ohki concluded that the correlation confirms the thermal model of these phenomena.

We can show, however, that their data are also consistent with a nonthermal model. If we observe free-free bremsstrahlung from an isothermal plasma with a temperature 
$T$ then the ratio of the $\mathrm{X}$-ray flux $F_{\mathrm{X}}$ to the radio flux $F_{\mathrm{R}}$ measured in the same units is:

$$
\frac{F_{\mathrm{X}}}{F_{\mathrm{R}}}=\exp \left\{\frac{E_{\mathrm{R}}-E_{\mathrm{X}}}{k T}\right\} \approx \exp -\left(\frac{E_{\mathrm{X}}}{k T}\right),
$$

where $E_{\mathrm{X}}$ and $E_{\mathrm{R}}$ are the $\mathrm{X}$-ray and radio energies and $E_{\mathrm{R}} \ll E_{\mathrm{X}}$. For $E_{\mathrm{X}} \approx 2 \mathrm{keV}$ and $k T \approx 2 \mathrm{keV}$ this yields a value of $F_{\mathrm{X}} / F_{\mathrm{R}} \approx 0.4$. Suppose on the other hand one were observing thin target bremsstrahlung given by the Bethe-Heitler formula (Jackson, 1962)

$$
F(\hbar \omega) \propto \ln \left[\frac{\left(E^{1 / 2}+(E-\hbar \omega)^{1 / 2}\right)^{2}}{\hbar \omega}\right],
$$

where $E$ is taken to be $4 \mathrm{keV}$ and $\omega$ is the observed frequency. Then $F_{\mathrm{X}} / F_{\mathrm{R}}$ is $\sim 0.1$, within a factor of 4 of the value given above for the thermal case. We have assumed the radio region is optically thin and have neglected free-bound emission. Thin target bremsstrahlung from a monoenergetic electron distribution would not give a thermal $\mathrm{X}$-ray spectrum but one could choose an incident electron spectrum which would result in an X-ray spectrum that would be consistent with a thermal spectrum as observed in broad band detectors. The point is that while the data of Hudson and Ohki are consistent with a thermal source for flares, they cannot be used to exclude possible nonthermal models.

\section{(b) NONTHERMAL ARGUMENTS}

Since the thermal interpretation for low energy X-ray events is so widely accepted, the only arguments for nonthermal emission have come from authors who have presented specific events which they felt warranted a nonthermal interpretation. We shall now discuss several such examples.

Blake and House (1971) analyzed iron line emission from a rocket crystal spectrometer flown in 1966, October 4. Their analysis of the intensity of the iron line complex at $1.9 \AA$ due to $\mathrm{K} \alpha$ line emission and the intensity of the optical $(2 \mathrm{p}-31)$ transitions of iron from 10 to $17 \AA$ yielded values of the ratio $F_{0} / F_{K}$ of less than 50 . They computed the expected ratio as a function of the electron temperature and found that an electron temperature of $200 \times 10^{6} \mathrm{~K}$ or a monoenergetic electron distribution peaked at $E \gtrsim 15 \mathrm{keV}$ was required to account for the observation. The emission measure of these electrons was calculated to be $\approx 3 \times 10^{47} \mathrm{~cm}^{-3}$. If we take a density of $n_{\mathrm{H}}=$ $=10^{9} \mathrm{~cm}^{-3}$, then the total energy of the quasithermal or nonthermal electrons is $\sim 7 \times 10^{30} \mathrm{erg}$, substantially larger than the energy of a small flare, estimated at $10^{29}$ erg (Lin, 1971). In view of the fact that there were no $\mathrm{H} \alpha$ flares, radio bursts or ionospheric disturbances reported at the time of the observations, their deductions would appear questionable.

Another event was analyzed by Landini et al. (1972). Their work with a small event in 1969 , January 7 observed with the 0.5 to $3 \AA$ and $1-8 \AA$ detectors on Solrad indicated that if the event were thermal the temperature first decreased early in the event and then increased late in the event, opposite to the results obtained by Horan and others. 
The authors interpreted this behavior as indicative of nonthermal emission. As in the event analyzed by Blake and House, there was no accompanying sign of nonthermal activity; however, because it was a small event none might be expected. The January 7 event occurred during the decay phase of an earlier, larger X-ray burst which may have presented problems for the data analysis. If such events do take place on the sun, one would like to see several more examples in order to define them as a class. Their existence constitutes proof of low energy nonthermal events only as long as one assumes that the cooling time is substantially longer than the heating time. If this is not the case, then the temperature and emission measure profiles may simply reflect the time variation of the heating mechanism.

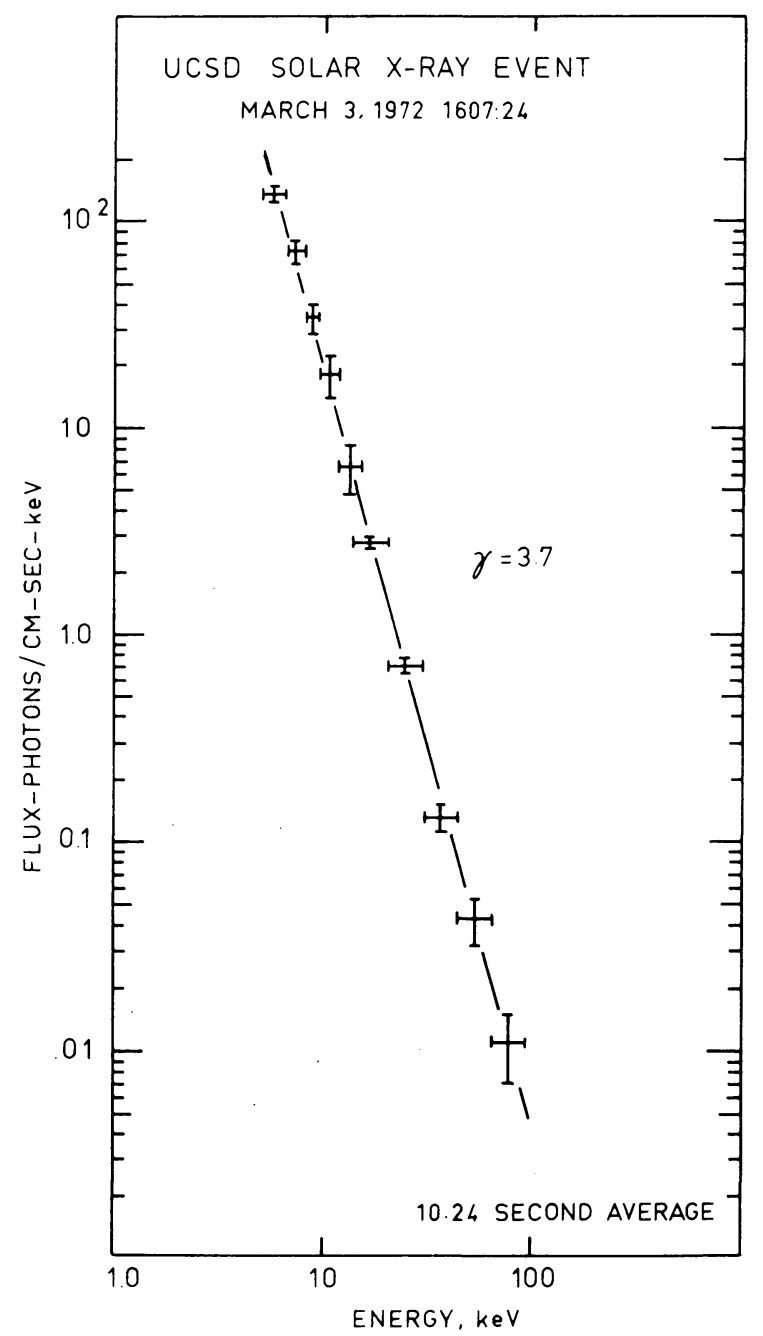

Fig. 9. The power-law spectrum at the peak of a hard X-ray burst observed on OSO 7 (Peterson et al., 1973). The power law fit extends from 5 to $100 \mathrm{keV}$. 
Peterson et al. (1973) have classified over 200 events observed on OSO-7 into four general categories. One of their categories, which consisted of only one event, has a single power law fit over both the hard and soft X-ray spectrum early in the event, followed by a steeper low energy spectrum. This event observed in 1972, March 3 could be fitted by a single power law from $5 \mathrm{keV}$ to $\sim 100 \mathrm{keV}$ with an exponent of $\gamma=3.7$, as shown in Figure 9. If we accept the nonthermal interpretation of the hard X-ray burst, then this event may indeed constitute evidence for the existence of low energy nonthermal bursts. The nature of the bremsstrahlung spectrum is such that it will
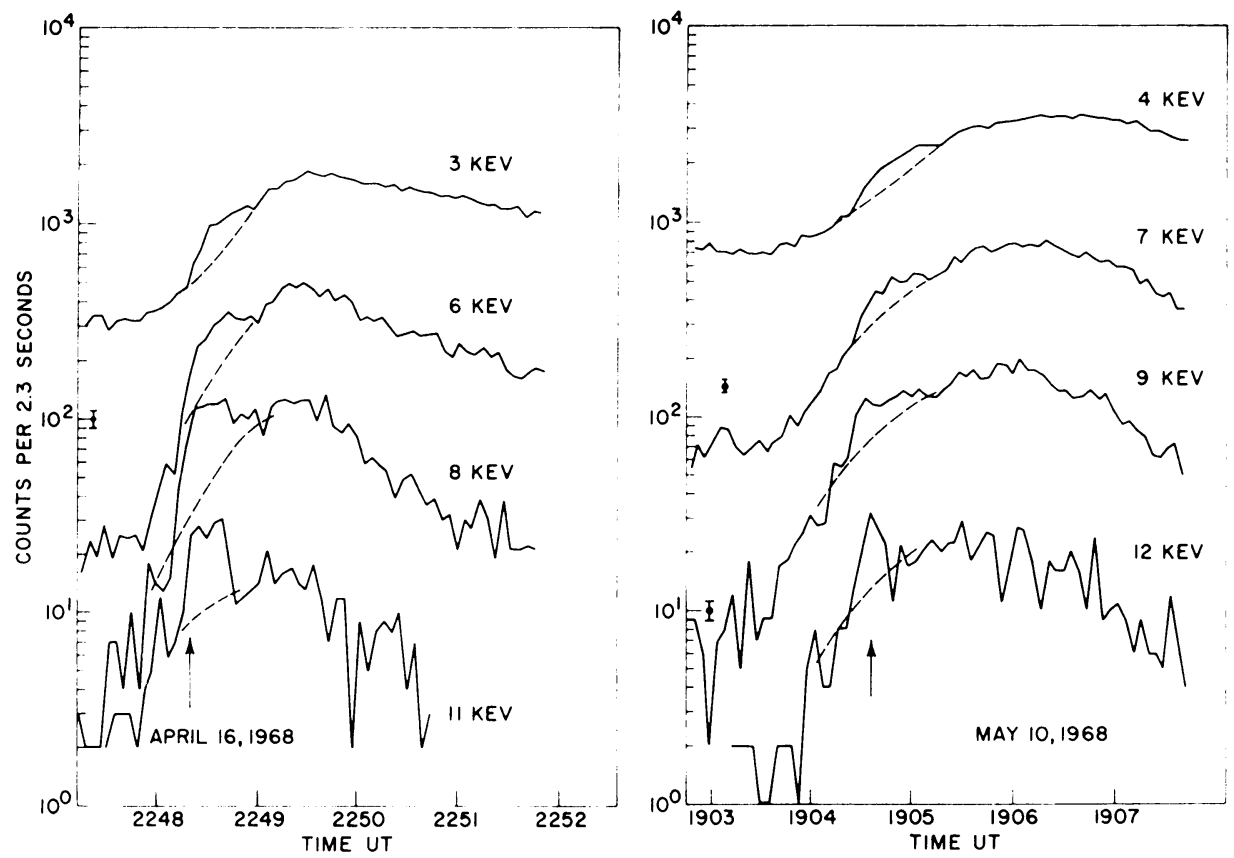

Fig. 10. Counting rate profiles of two impulsive events from the NRL detector on OGO 5 (Kahler and Kreplin, 1971). The dashed lines represent the estimated profiles of the thermal components during the impulsive bursts.

extend down to the lowest measurable energy for any electron spectrum. The rarity of the March 3 event may simply be due to the requirement that the development of the low energy thermal event be sufficiently retarded in time relative to the impulsive burst for the low energy end of the impulsive burst to dominate the spectrum. Kahler and Kreplin (1971) found that on only two out of twelve impulsive hard X-ray events could the impulsive component be traced down to $3 \mathrm{keV}$. In the other ten cases the slowly varying component dominated the low energy spectrum. In their analysis they assumed that they could accurately estimate the intensity-time profile of the $E<10 \mathrm{keV}$ slowly varying fluxes and subtract those profiles from the profiles of the total emission to yield the profiles of the impulsive component alone. Figure 10 shows the flux profiles of these two events. The fact that Peterson et al. and Kahler and Kreplin both 

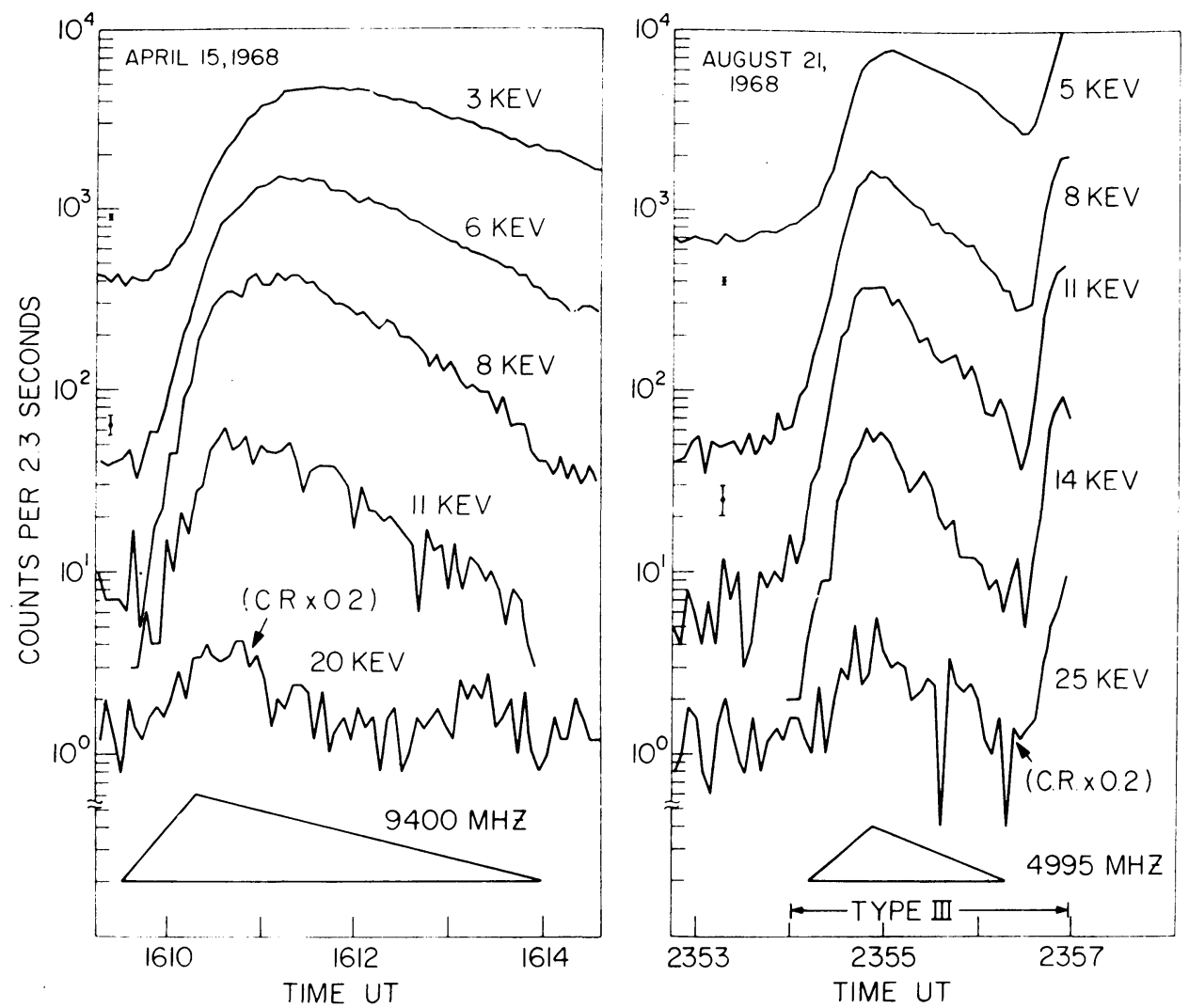

Fig. 11. Two low energy impulsive events which were accompanied by impulsive events in the $E>20 \mathrm{keV}$ range and were closely correlated with microwave bursts (Kahler, 1973).

were able to trace the low energy end of the impulsive bursts down to $5 \mathrm{keV}$ using different analytical methods seems strong evidence that in at least some cases the low energy emission is dominated by a nonthermal component. This statement, of course, assumes a nonthermal interpretation for the impulsive burst.

Kahler (1973) has used the method of tracing the intensity-time profiles down to low energies to argue that three events he observed on OGO 5 in 1968 might be purely nonthermal in nature down to $3 \mathrm{keV}$. In each case an impulsive hard X-ray burst with an accompanying microwave burst could be traced down to $3 \mathrm{keV}$ where only a single simple rise and fall profile was seen, as shown in Figure 11. He pointed out, however, that the low energy profiles were quite consistent with the behavior usually seen in slowly varying bursts which are almost always treated as thermal.

(c) SPECTRAL INTERPRETATIONS

Kahler and Kreplin (1971) have discussed the ambiguity involved in the interpretation of low energy continuum spectra. One can measure the slope of the continuum at some energy $E$ and interpret it either as a power law spectrum of exponent 
$\gamma$ or as a thermal spectrum of temperature $T$. The two are related by the equation

$$
k T=E /(\gamma-1) \text {. }
$$

Using reasonable values of $\gamma$ such as those obtained by Kane (1971) and $2 \lesssim E \lesssim 8 \mathrm{keV}$, the corresponding temperatures range from 7 to $55 \times 10^{6} \mathrm{~K}$, in agreement with the temperatures one usually obtains from various broadband and spectrometer measurements. The time behavior of the slope of the continuum also provides no help in distinguishing between power law and thermal interpretations since the spectra typically first get harder and then near the peak begin to get softer again.

One feature of the low energy spectrum which might be used for nonthermal criteria is the line emission observed with crystal spectrometers. Landini et al. (1973) have performed calculations of the ratios of $\mathrm{H}$-like to $\mathrm{He}$-like ions for a plasma with a power law electron spectrum. They assumed a quasi-steady state situation in which the electrons were able to maintain a power law distribution in time and calculated the line ratios for various power law spectral exponents and low energy cutoffs in the electron distributions. It was their conclusion that there was no possibility of distinguishing between the thermal and nonthermal interpretation by using this method. Figure 12 shows a comparison of published data with their calculations for the case with a cutoff of $E_{1}=1 \mathrm{keV}$. Since lower values of the cutoff imply lower values of $\gamma$

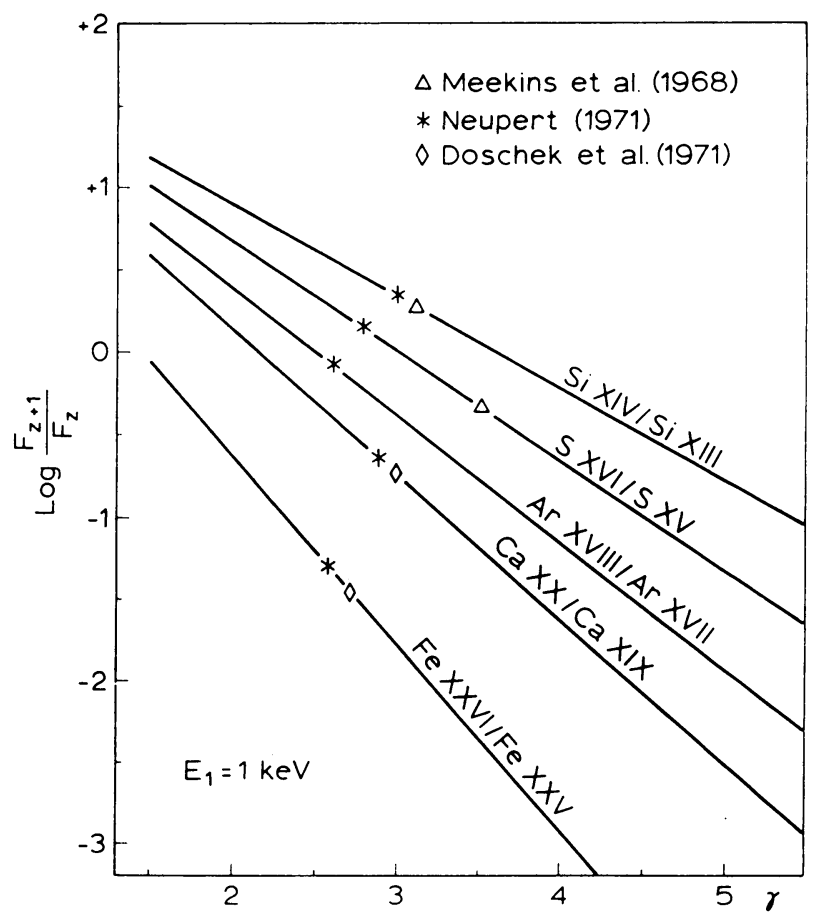

Fig. 12. Computed flux ratios between the resonance lines of $\mathbf{H}$-like and He-like ions as a function of the electron power law spectral index $\gamma$ (Landini et al., 1973). A low energy cutoff of $1 \mathrm{keV}$ was used. Some experimental values are shown for comparison. 
than those derived by Kane (1971) for higher energies, Landini et al. (1973) suggest that a higher energy cutoff with associated larger values of $\gamma$ represent a more reasonable interpretation of the data.

A more obvious method is to look for enhanced $\mathrm{K} \alpha$ line emission either during the impulsive burst or during the slowly-varying component. Phillips and Neupert (1973) have calculated the $\mathrm{K} \alpha$ line emission expected from $\mathrm{S}$, $\mathrm{Ar}, \mathrm{Ca}$, and $\mathrm{Fe}$ for both the thermal and nonthermal cases. The electron spectra deduced by Kane and Anderson (1970) for impulsive bursts were used for the nonthermal case, but it was found that in most cases the expected line emission was relatively weak. Phillips and Neupert did their nonthermal calculations for an assumed ion temperature of $2 \times 10^{6} \mathrm{~K}$. They point out that the proportions of $\mathrm{S}, \mathrm{Ar}$, and $\mathrm{Ca}$ atoms ionized to the B-like stage or lower, which are necessary for $\mathrm{K} \alpha$ line emission in the solar atmosphere, are very small at temperatures above $10 \times 10^{6} \mathrm{~K}$, hence no significant $\mathrm{K} \alpha$ line emission would be expected. It would appear, then, that the requirement of low ion temperatures and energetic electrons for $\mathrm{K} \alpha$ line emission would imply a possible nonthermal event whenever the electron temperatures deduced from the continuum measurements were high but.yet $\mathrm{K} \alpha$ line emission was simultaneously detected. This procedure would be more useful in working with the hard impulsive bursts than with low energy events.

\section{(d) ANISOTROPY AND POLARIZATION RESULTS}

As discussed in Section II, one expects to see a heliographic longitude dependence of the number of observed soft X-ray bursts for certain nonthermal models. The 2-12 $\AA$ X-ray bursts observed with the University of Iowa Geiger counter experiments on Explorers 33 and 35 were used by Pinter (1969) and Ohki (1969) to obtain the longitude dependence of the bursts. Ohki found no longitude dependence of a set of 232 such bursts, but Pinter found a peak at $30-40^{\circ}$ longitude using a larger sample of 490 bursts. Neither author took into account the $\mathrm{H} \alpha$ flare longitude distribution.

Drake (1971) plotted the longitude distribution of a total of $26982-12 \AA$ bursts and took into account the $\mathrm{H} \alpha$ flare distribution for the period he studied. His result is shown in Figure 13. No statistically significant directivity is indicated with the possible exception of the $70^{\circ}$ to $90^{\circ}$ region. The results in this region are due to the fact that the $\mathrm{H} \alpha$ distribution during the time of X-ray coverage had a sharp drop in the $70^{\circ}-$ $80^{\circ}$ longitude region followed by a peak in the $80^{\circ}$ to $90^{\circ}$ region. The $2-12 \AA$ data should not be subject to substantial modification through the Compton backscattering process (Tomblin, 1972) because of the dominance of photoelectric absorption over Compton scattering. The lack of directivity can be taken as evidence for a thermal interpretation of the bulk of soft X-ray events.

Unfortunately, there do not appear to be any polarization measurements made on the low energy X-ray events probably due partly to the assumed thermal nature of these events. Brown (1974) has pointed out that Compton scattering will introduce a small amount of polarization in the detected signal even if the source is thermal. Wolff (1973) has calculated the minimum polarization measurable over a $100 \mathrm{~s}$ period with a lithium scattering block experiment as a function of energy for a flare spectrum. 


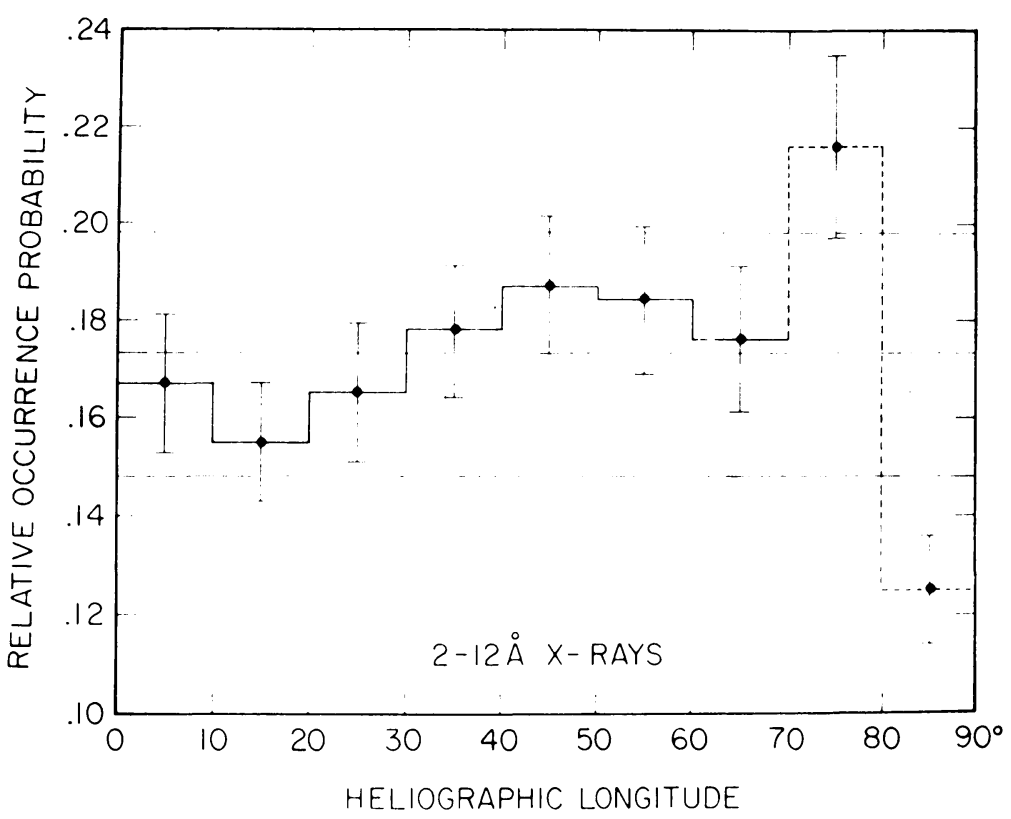

Fig. 13. The relative probability for the occurrence of $2-12 \AA$ solar X-ray bursts as a function of solar longitude (Drake, 1971). Data are from Explorers 33 and 35 from July 1966 to September 1968.

The detectable polarization is very low $(<1 \%)$ in the lowest energy range $(5-9 \mathrm{keV})$ and increases with energy due to the poorer statistics at high energy. Polarization measurements in the low energy X-ray range are facilitated by higher fluxes and longer characteristic time scales than occur with the high energy impulsive bursts. Two polarization experiments are planned for the Solrad 11 satellite to be launched in 1975, a Bragg crystal mounted to observe a region of the continuum at $2.8 \AA$ and a Compton scattering experiment with two energy channels at about 8 to 15 and 15 to $50 \mathrm{keV}$. The experiments also have the advantage that they are mounted around the spin axis of the spacecraft which points at the Sun. This experiment should provide measurements very useful in establishing the thermal or nonthermal nature of low energy X-ray events.

\section{References}

Arnoldy, R. L., Kane, S. R., and Winckler, J. R.: 1968, Astrophys. J. 151, 711.

Blake, R. L. and House, L. L. : 1971, Astrophys. J. 166, 423.

Brown, J. C.: 1972, Solar Phys. 26, 441.

Brown, J. C.: 1974, in G. Newkirk (ed.), 'Coronal Disturbances', IAU Symp. 57, D. Reidel Publ. Co., Dordrecht, Holland, p. 395.

Brown, J. C., McClymont, A. N., and McLean, I. S.: 1974, Nature 247, 448.

Chubb, T. A.: 1970, in E. R. Dyer (General Ed.), Solar Terrestrial Physics, Vol. 1, D. Reidel Publ. Co., Dordrecht, Holland, p. 99.

Culhane, J. L. and Phillips, K. J. H.: 1970, Solar Phys. 11, 117.

Datlowe, D. W., Elcan, M. J., and Hudson, H. S.: 1974, Solar Phys. 39, 155

de Jager, C. and Kundu, M. R.: 1963, Space Res. 3, 836. 
Doschek, G. A., Meekins, J. F., Kreplin, R. W., Chubb, T. A., and Friedman, H. : 1972, in K. Schindler (ed.), Cosmic Plasma Physics, Plenum, p. 165.

Drake, J. F.: 1971, Solar Phys. 16, 152.

Elwert, G. and Haug, E.: 1971, Solar Phys. 20, 413.

Frost, K. J.: 1969, Astrophys. J. 158, L159.

Frost, K. J. and Dennis, B. R.: 1971, Astrophys. J. 165, 655.

Hudson, H. S. and Ohki, K.: 1972, Solar Phys. 23, 155.

Jackson, J. D.: 1962, Classical Electrodynamics, Wiley, New York.

Kahler, S.: 1971a, Astrophys. J. 164, 365.

Kahler, S.: 1971b, Astrophys. J. 168, 319.

Kahler, S. W.: 1973, in R. Ramaty and R. G. Stone (eds.), High Energy Phenomena on the Sun, NASA SP-342, p. 124.

Kahler, S. W. and Kreplin, R. W.: 1971, Astrophys. J. 168, 531.

Kahler, S. W., Meekins, J. F., Kreplin, R. W., and Bowyer, C. S.: 1970, Astrophys. J. 162, 293.

Kane, S. R.: 1971, Astrophys. J. 170, 587.

Kane, S. R.: 1972, Solar Phys. 27, 174.

Kane, S. R.: 1973a, in R. Ramaty and R. G. Stone (eds.), High Energy Phenomena on the Sun, NASA SP-342, p. 55.

Kane, S. R.: 1973b, in G. Newkirk (ed.), 'Coronal Disturbances', IAU Symp. 57, D. Reidel Publ. Co., Dordrecht, Holland, p. 105.

Kane, S. R.: 1975 (to be published).

Kane, S. R. and Anderson, K. A.: 1970, Astrophys. J. 162, 1003.

Landini, M., Monsignori Fossi, B. C., and Pallavicini, R.: 1972, Solar Phys. 27, 164.

Landini, M., Monsignori Fossi, B. C., and Pallavicini, R.: 1973, Solar Phys. 29, 93.

Lin, R. P.: 1971, Acceleration of 10-100 keV Electrons in Solar Flares, Seminar on the Acceleration of Particles in Near-Earth and Interplanetary Space, Galaxy, and Metagalaxy, Leningrad.

Lin, R. P., Evans, L. G., and Fainberg, J.: 1973, Astrophys. Letters 14, 191.

Milkey, R. W.: 1971, Solar Phys. 16, 465.

Ohki, K.: 1969, Solar Phys. 7, 260.

Parks, G. K. and Winckler, J. R.: 1969, Astrophys. J. 155, L117.

Peterson, L. E., Datlowe, D. W., and McKenzie, D. L.: 1973, in R. Ramaty and R. G. Stone (eds.), High Energy Phenomena on the Sun, NASA SP-342, p. 132.

Phillips, K. J. H.: 1973, Observatory 93, 17.

Phillips, K. J. H. and Neupert, W. M.: 1973, Solar Phys. 32, 209.

Pinter, S.: 1969, Solar Phys. 8, 142.

Ramaty, R.: 1973, in R. Ramaty and R. G. Stone (eds.), High Energy Phenomena on the Sun, NASA SP-342, p. 188.

Santangelo, N., Horstman, H., and Horstman-Moretti, E.: 1973, Solar Phys. 29, 143.

Shaw, M. L.: 1972, Solar Phys. 27, 436.

Sommerfeld, A. J. F.: 1951, Atombau und Spectrallinien, Ungar, New York.

Spitzer, L.: 1962, Physics of Fully Ionized Gases, Interscience, New York.

Sturrock, P. A.: 1973, in R. Ramaty and R. G. Stone (eds.), High Energy Phenomena on the Sun, NASA SP-342, p. 3.

Takakura, T. and Kai, K.: 1966, Publ. Astron. Soc. Japan 18, 57.

Takakura, T., Ohki, K., Shibuya, N., Fujii, M., Matsuoka, M., Miyamoto, S., Nishamura, J., Oda, M., Ogawara, Y., and Ota, S.: 1971, Solar Phys. 16, 454.

Tindo, I. P., Ivanov, V. D., Mandel'stam, S. L., and Shuryghin, A. I.: 1972a, Solar Phys. 24, 429.

Tindo, I. P., Ivanov, V. D., Valniček, B., and Livshits, M. A.: 1972b, Solar Phys. 27, 426.

Tindo, I. P., Mandel'stam, S. L., and Shuryghin, A. I.: 1973, Solar Phys. 32, 469.

Tomblin, F. F.: 1972, Astrophys. J. 171, 377.

Wolff, R. S. : 1973, in R. Ramaty and R. G. Stone (eds.), High Energy Phenomena on the Sun, NASA SP-342, p. 162. 